\title{
Erratum to: A novel bedside test for ACPA: the CCPoint test is moving the laboratory to the rheumatologist's office
}

Gisele Zandman-Goddard $^{1,2,5} \cdot$ Alessandra Soriano $^{1,4} \cdot$ Boris Gilburd $^{\mathbf{1}}$. Merav Lidar ${ }^{2,3}$ - Shaye Kivity ${ }^{2,3}$ - Ron Kopilov ${ }^{1}$ - Pnina Langevitz ${ }^{2,3}$. Yehuda Shoenfeld ${ }^{1,6} \cdot$ Nancy Agmon-Levin ${ }^{1,2}$

Published online: 13 September 2016

(C) Springer Science+Business Media New York 2016

\section{Erratum to: Immunol Res}

\section{DOI 10.1007/s12026-016-8846-2}

The original version of this article unfortunately contained a mistake. The given name and family name of the first author were incorrect. The author name has been corrected in this erratum.

The online version of the original article can be found under doi: 10 . 1007/s12026-016-8846-2.

Nancy Agmon-Levin

nancy.agmon-levin@sheba.health.gov.il

1 Zabludowicz Center for Autoimmune Diseases, Chaim Sheba Medical Center, Tel-Hashomer 52621, Israel

2 Sackler School of Medicine, Tel Aviv University, Tel Aviv, Israel

3 The Zabludowicz Center for Autoimmune Diseases, Rheumatology Unit, Sheba Medical Center, Tel-Hashomer 52621, Israel

4 Department of Clinical Medicine and Rheumatology, Campus Bio-Medico University, Rome, Italy

5 Department of Medicine C, Wolfson Medical Center, Holon, Israel

6 Incumbent of the Laura Schwarz-Kipp Chair for Research of Autoimmune Diseases, Sackler Faculty of Medicine,

Tel-Aviv University, Tel-Aviv, Israel 